ELECTRONIC RESEARCH ANNOUNCEMENTS OF THE AMERICAN MATHEMATICAL SOCIETY

Volume 6, Pages 21-30 (March 28, 2000)

S $1079-6762(00) 00076-7$

\title{
MATING QUADRATIC MAPS WITH KLEINIAN GROUPS VIA QUASICONFORMAL SURGERY
}

\author{
S. R. BULLETT AND W. J. HARVEY
}

(Communicated by Svetlana Katok)

\begin{abstract}
Let $q: \hat{\mathbb{C}} \rightarrow \hat{\mathbb{C}}$ be any quadratic polynomial and $r: C_{2} * C_{3} \rightarrow$ $\operatorname{PSL}(2, \mathbb{C})$ be any faithful discrete representation of the free product of finite cyclic groups $C_{2}$ and $C_{3}$ (of orders 2 and 3 ) having connected regular set. We show how the actions of $q$ and $r$ can be combined, using quasiconformal surgery, to construct a $2: 2$ holomorphic correspondence $z \rightarrow w$, defined by an algebraic relation $p(z, w)=0$.
\end{abstract}

\section{INTRODUCTION}

Given two abstractly isomorphic Fuchsian groups $G_{1} \subset P S L(2, \mathbb{R})$ and $G_{2} \subset$ $P S L(2, \mathbb{R})$, acting on the upper and lower halves $\mathcal{U}$ and $\mathcal{L}$ of the complex plane respectively, each having limit set $\hat{\mathbb{R}}=\mathbb{R} \cup \infty$, and such that the action of $G_{1}$ on $\hat{\mathbb{R}}$ is topologically conjugate to that of $G_{2}$, it is well known that one can mate the actions of $G_{1}$ and $G_{2}$ to obtain a Kleinian group $G \subset P S L(2, \mathbb{C})$, isomorphic as an abstract group to both $G_{1}$ and $G_{2}$, such that the limit set $\Lambda$ of $G$ is a simple closed (fractal) curve and the actions of $G$ on the two components of $\Omega=\hat{\mathbb{C}}-\Lambda$ are conformally conjugate to those of $G_{1}$ on $\mathcal{U}$ and $G_{2}$ on $\mathcal{L}$.

Equally, given two polynomial maps $P$ and $Q$ of the same degree $n$, having connected filled Julia sets $K(P)$ and $K(Q)$ respectively, it is well known that in certain cases one can mate the actions to obtain a rational map $R$ such that the complement $\Omega$ of the Julia set $J(R)$ is a disjoint union of two open sets, on one of which the action of $R$ is conformally conjugate to that of $P$ on the interior $K(P)^{\circ}$ of its filled Julia set, and on the other of which the action of $R$ is conformally conjugate to that of $Q$ on $K(Q)^{\circ}$. A necessary condition for a mating of two quadratic polynomials $P: z \rightarrow z^{2}+c$ and $Q: z \rightarrow z^{2}+c^{\prime}$ to exist is that $c$ and $c^{\prime}$ should not belong to conjugate limbs of the connectivity locus in parameter space: this was first shown also to be a sufficient condition in the case that $P$ and $Q$ are postcritically finite [12, and subsequently for more general classes of $P$ and $Q$.

It is also possible to mate certain Kleinian groups with polynomial maps. To realise such matings we have to move into the larger world of holomorphic correspondences. A holomorphic correspondence, of bidegree $m: n$, on the Riemann sphere $\hat{\mathbb{C}}$, is a multivalued map $z \rightarrow w$ defined by a relation $p(z, w)=0$, where $p$ is

Received by the editors December 22, 1999.

2000 Mathematics Subject Classification. Primary 37F05; Secondary 30D05, 30F40, 37 F30.

Key words and phrases. Holomorphic dynamics, quadratic maps, Kleinian groups, quasiconformal surgery, holomorphic correspondences. 


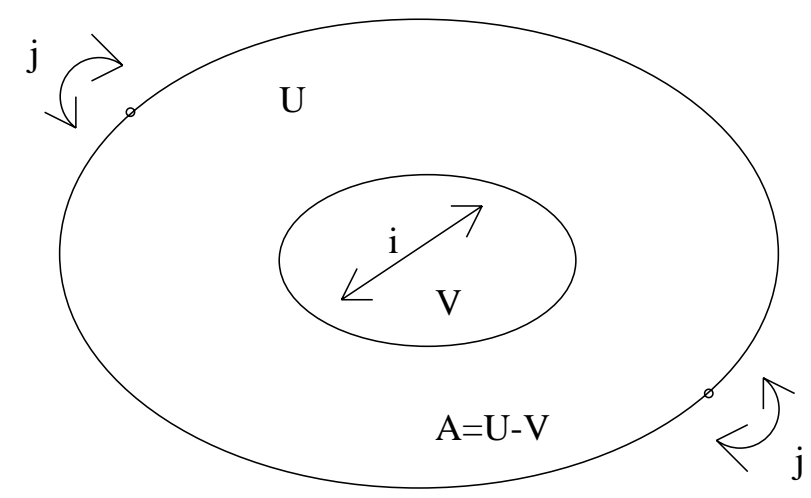

Figure 1. An annulus $A$ for the quadratic map $q: z \rightarrow z^{2}+c$.

a polynomial of degree $m$ in $z$ and $n$ in $w$. We require that $p(z, w)$ has no square factors, so that a generic point $w$ has $m$ inverse images $z$ and a generic point $z$ has $n$ images $w$. Equivalently, a holomorphic correspondence on $\hat{\mathbb{C}}$ is defined by a (singular) Riemann surface in $\hat{\mathbb{C}} \times \hat{\mathbb{C}}$ with the two projections to $\hat{\mathbb{C}}$ branched-coverings of degrees $m$ and $n$ respectively. Examples of holomorphic correspondences are rational maps (defined by $P(z)-w=0$ ), their inverses (defined by $P(w)-z=0$ ), and finitely generated Kleinian groups (defined by $\left(w-A_{1} z\right)\left(w-A_{2} z\right) \cdots\left(w-A_{n} z\right)=0$, where $A_{1}, \ldots, A_{n}$ are Möbius transformations generating the group in question). We formulate below (in Section 3) what it means to say that a holomorphic correspondence is a mating of a particular Kleinian group and a particular polynomial map. The first examples were described in [3] and more general constructions were presented in [5]. These examples and constructions pick out particular classes of polynomial relations $p(z, w)=0$ and then in appropriate circumstances identify the resulting correspondences as matings. Below we show how it is possible to create a mating of a quadratic map and a representation of the group $C_{2} * C_{3}$ to order, by fitting the pieces together using quasiconformal surgery [8]. The key observation that enables us to get started (Section 4.1 below) is that a certain 'pair of pants' domain associated to a representation of $C_{2} * C_{3}$ double covers an annulus carrying precisely the same combinatorial data as does a 'fundamental annulus' for a quadratic-like map.

\section{THE INGREDIENTS}

2.1. The quadratic map. Given any quadratic map $q: z \rightarrow z^{2}+c$, there is a holomorphic conjugacy from $z \rightarrow z^{2}$ to $q$ on a neighbourhood of $\infty$, fixing the point $\infty$ and tangent to the identity map there [7. An equipotential for $q$ is the image of a circle $\left\{R e^{2 \pi i t}: 0 \leq t<1\right\}$ under this conjugacy. It is a smooth Jordan curve parametrised by external angle $t$. The region bounded by such an equipotential is a simply-connected domain $V$, mapped $2: 1$ by $q$ onto a larger domain $U \supset V$ which also has boundary an equipotential parametrised by external angle (the restriction $q: V \rightarrow U$ is an example of a quadratic-like map in the sense of Douady and Hubbard [8]). We shall denote the annulus $U-V$ by $A$ and its inner and outer boundaries by $\partial_{1} A$ and $\partial_{2} A$ respectively (Figure 1 ). The map $q$ sends $\partial_{1} A$ two-toone onto $\partial_{2} A$. For future reference we note the existence of an involution $i: z \rightarrow-z$ 


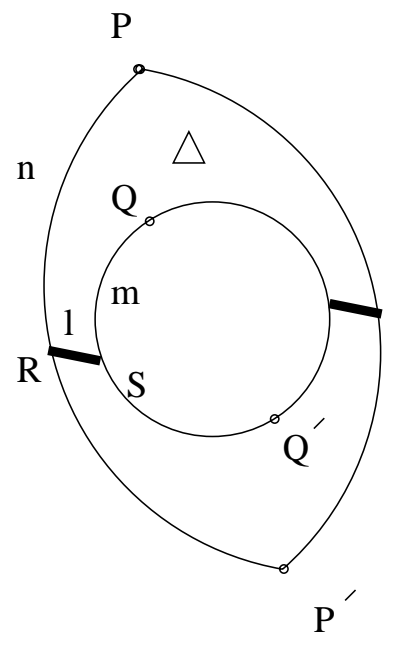

Figure 2. A fundamental domain $\Delta$ for the group $G=\langle\sigma, \rho, \chi\rangle$.

on $V$ sending each $z \in V$ to the other point which has the same image in $U$ under $q$, and an involution $j$ on $\partial_{2} A$ given by $t \rightarrow 1-t$ on external angles (in fact in what follows $j$ may be taken to be any smooth orientation-reversing involution on $\partial_{2} A$ ).

For simplicity, until the final section of this article we shall assume that the filled Julia set $K(q)$ is connected. The corresponding set $\mathcal{M}$ of values of the parameter $c$ is known as the connectivity locus or Mandelbrot set.

2.2. The Kleinian group. Up to conjugacy each representation $r$ of $C_{2} * C_{3}$ in $P S L(2, \mathbb{C})$ is determined by a single complex parameter, the cross-ratio between the fixed points on $\hat{\mathbb{C}}$ of the action of the generator $\sigma$ of $C_{2}$ and those of the generator $\rho$ of $C_{3}$. Such a representation comes equipped with a (unique) involution $\chi$ which exchanges the two fixed points of $\sigma$ and also those of $\rho$, so that $\chi \sigma=\sigma \chi$ and $\chi \rho=\rho^{-1} \chi[4]$. On the Poincaré 3 -disc $\chi$ is simply rotation through $\pi$ around the common perpendicular to the axes of $\sigma$ and $\rho$. Write $G$ for the group $\langle\sigma, \rho, \chi\rangle$.

The faithful discrete actions $r$ with connected regular set $\Omega(G)$ form a single quasiconformal conjugacy class, the class of representations for which one can find simply-connected fundamental domains for $\sigma$ and $\rho$ with interiors together covering the whole Riemann sphere (i.e. the conditions of the simplest form of the Klein Combination Theorem are satisfied) [9, 10. Such fundamental domains may be constructed as illustrated in Figure 2. Here $P$ and $P^{\prime}$ are the fixed points of $\rho, Q$ and $Q^{\prime}$ are the fixed points of $\sigma, R$ is a fixed point of (the involution) $\chi \rho$ and $S$ is a fixed point of $\chi \sigma$. The lines $l, m$ and $n$, joining $R$ to $S, Q$ to $S$ and $R$ to $P$, are chosen such that they are smooth and remain non-intersecting in the quotient orbifold $\Omega(G) / G$. The region bounded by $n, \rho n, \chi n$ and $\chi \rho n$ is a fundamental domain for $\rho$, and the region exterior to the loop made up of $m, \sigma m, \chi m$ and $\chi \sigma m$ is a fundamental domain for $\sigma$. The intersection of these two regions is a fundamental domain for the (faithful) action of $C_{2} * C_{3}$ on $\Omega(G)$, and the half $\Delta$ of this intersection bounded by $n, l, m, \sigma m, \chi l$ and $\rho n$ is a fundamental domain for the action of $G$. The union of all translates of $\Delta$ under elements of $C_{2} * C_{3}$ is a 
topological disc $D$ which is a fundamental domain for the action of $\chi$ on $\Omega(G)$. The complement $\Lambda(G)$ of $\Omega(G)=D \cup \chi(D)$ in $\hat{\mathbb{C}}$ is a Cantor set.

\section{The Definition of a mating and the statement of the Theorem}

We say that a $2: 2$ holomorphic correspondence $f: \hat{\mathbb{C}} \rightarrow \hat{\mathbb{C}}$ is a mating of the quadratic map $q: z \rightarrow z^{2}+c$ (where $c \in \mathcal{M}$ ) and the faithful discrete representation $r$ of $C_{2} * C_{3}$ (having connected regular set) if $\hat{\mathbb{C}}$ is partitioned into an open set $\Omega$ and a closed set $\Lambda$, each completely invariant under $f$ and with the following properties:

(I) $\Lambda$ is the disjoint union of two sets $\Lambda_{+}$and $\Lambda_{-}$, on which the $2: 2$ correspondence $f: \Lambda \rightarrow \Lambda$ decomposes into the following parts:

(i) $f: \Lambda_{-} \rightarrow \Lambda_{-}$, a $2: 1$ correspondence (a map of degree two);

(ii) $f: \Lambda_{+} \rightarrow \Lambda_{+}$, a $1: 2$ correspondence (the inverse of a map of degree two);

(iii) $f: \Lambda_{-} \rightarrow \Lambda_{+}$, a $1: 1$ correspondence (a bijection).

(II) There is a homeomorphism from the filled Julia set $K$ of $q$ to $\Lambda_{-}$conjugating $\left.q\right|_{K}$ to $\left.f\right|_{\Lambda_{-}}$and a homeomorphism from $K$ to $\Lambda_{+}$conjugating $\left.q\right|_{K}$ to $\left.f^{-1}\right|_{\Lambda_{+}}$. Both are conformal on the interior of $K$.

(III) The $2: 2$ correspondence $f: \Omega \rightarrow \Omega$ acts properly discontinuously and there is a conformal homeomorphism $h$ from the orbifold $\Omega / f$ to the orbifold $\Omega(G) / G$ compatible with the actions of $f$ and $G$ respectively, in the following sense: there exist a (completely invariant) set of curves $\mathcal{C}$ in $\Omega$ and a fundamental domain $D$ for the action of $\chi$ on $\Omega(G)$ which is invariant under $C_{2} * C_{3}$, such that $h$ lifts to a conformal homeomorphism $(\Omega-\mathcal{C}) \rightarrow D$ conjugating $f$ to $\left\{\sigma \rho, \sigma \rho^{-1}\right\}$.

Theorem 1. For every quadratic map $q: z \rightarrow z^{2}+c$ with $c \in \mathcal{M}$ and every faithful discrete representation $r$ of $C_{2} * C_{3}$ in $P S L(2, \mathbb{C})$ having connected regular set, there exists a polynomial relation $p(z, w)=0$ defining a $2: 2$ correspondence $z \rightarrow w$ which is a mating of $q$ with $r$.

A modification dealing with the case when $c$ is outside $\mathcal{M}$ will be outlined in the final section of this paper.

\section{The Construction And the proof of the Theorem}

4.1. An annulus associated to the Kleinian group, and a $2: 2$ correspondence on it. The quotient orbifold $\Omega(G) / G$ is the Riemann surface (with cone point singularities) obtained from $\Delta$ by making the boundary identifications corresponding to $\rho, \sigma$ and $\chi$. Covering this orbifold we have an annulus $B$ consisting of three contiguous copies of $\Delta$ in $\Omega(G)$, namely $\Delta \cup \rho \Delta \cup \rho^{-1} \Delta$, with the boundary identifications (induced by $\chi$ ) indicated in Figure 3 . Concretely we may regard $B$ as a subset of the quotient Riemann sphere $\hat{\mathbb{C}} / \chi$. We remark that $\Delta \cup \rho \Delta \cup \rho^{-1} \Delta$ is itself a fundamental domain for the action of the index three subgroup $\langle\chi, \sigma, \rho \sigma \rho\rangle$ of $G$, and that $\Delta \cup \rho \Delta \cup \rho^{-1} \Delta$ and its image under $\chi$ make up a 'pair of pants' fundamental domain for $\langle\sigma, \rho \sigma \rho\rangle$, the annulus $B$ being the quotient of this 'pair of pants' by the action of $\chi$.

The rotation $\rho$ maps $\Delta \cup \rho \Delta \cup \rho^{-1} \Delta$ to itself in the obvious way, with a single fixed point at $P$, but since $\rho$ anticommutes with $\chi$, this action does not descend to a rotation on the quotient annulus $B$. Rather, the action of the pair $\left\{\rho, \rho^{-1}\right\}$ on $\Delta \cup \rho \Delta \cup \rho^{-1} \Delta$ descends to the action of a $2: 2$ correspondence $g$ on $B$. Under this 

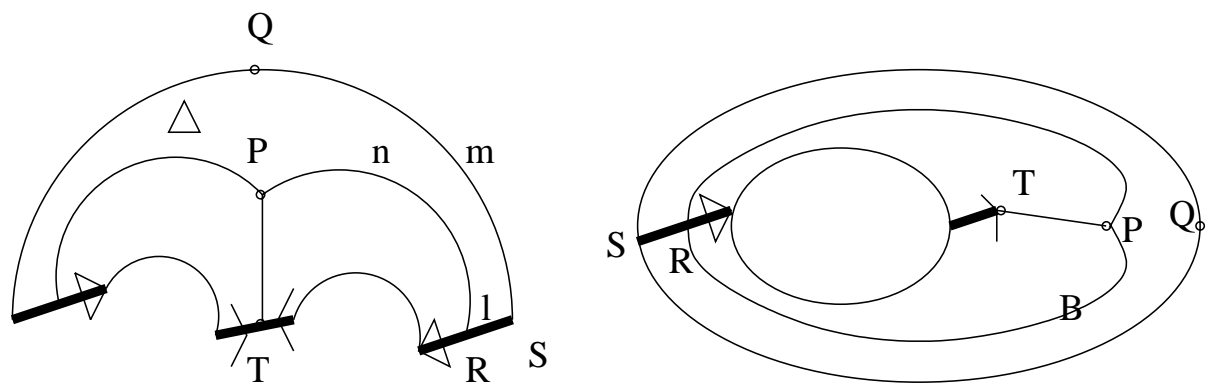

Figure 3. The set $\left(\Delta \cup \rho \Delta \cup \rho^{-1} \Delta\right)$ and its quotient, the annulus $B$.

$2: 2$ correspondence each $z \in B$ is mapped to the points $\rho z$ and $\rho^{-1} z$ (or rather to their equivalence classes under the action of $\chi$ ). The points $P$ and $T$ are singular for $g$, having unique images $P$ and $R$, respectively, but all other points of $B$ have two distinct images under $g$, and also 2 distinct inverse images, since $g=g^{-1}$. Note that in a neighbourhood of $P$ the correspondence $g$ behaves like a pair of rotations through $2 \pi / 3$ and $-2 \pi / 3$, but in a neighbourhood of $T$ it behaves like a square root map. Generic orbits of $g$ have cardinality three, the correspondence $g$ sending each point of an orbit to the other two, and the image of $\Delta$ in $B$ is a 'fundamental domain' for the action. The boundary of $B$ is divided into three segments (two inner and one outer, Figure 3), each of which is mapped to the other two by $g$. Thus when its domain is restricted to the inner boundary $\partial_{1} B$, and its range is restricted to the outer boundary $\partial_{2} B$, the correspondence $g$ defines a two to one map. When restricted to a correspondence from the inner boundary to itself, $g$ defines a (fixed point free) bijection. Moreover, since the involution $\sigma$ commutes with $\chi$, it descends to an involution (which we shall also denote $\sigma$ ) on the outer boundary $\partial_{2} B$ of $B$, having fixed points $Q$ and $S$. Observe that since the composition $\sigma \circ g: \partial_{1} B \rightarrow \partial_{2} B$ is an orientation preserving two to one map, and the bijection $g: \partial_{1} B \rightarrow \partial_{1} B$ is the covering involution of this map, the annulus $B$ carries the same data as that furnished on the annulus $A$ by the quadratic map $q$.

4.2. A bijection between the annuli $A$ and $B$. In general the annuli $A$ and $B$ will not be conformally equivalent: the conformal equivalence class of an annulus is determined by its modulus, a positive real number [2]. However, any two annuli are quasiconformally equivalent.

Lemma 1. There exists a quasiconformal homeomorphism $h$ from $A$ to $B$ which restricts to a smooth homeomorphism from $\partial A$ to $\partial B$ conjugating the boundary maps $\left(q: \partial_{1} A \rightarrow \partial_{2} A, j: \partial_{2} A \rightarrow \partial_{2} A\right)$ to the boundary maps $\left(\sigma \circ g: \partial_{1} B \rightarrow\right.$ $\left.\partial_{2} B, \sigma: \partial_{2} B \rightarrow \partial_{2} B\right)$.

Proof. We first use the fixed points of $j$ to divide the outer boundary $\partial_{2} A$ of $A$ into two intervals and choose any smooth homeomorphism $h$ from one of these intervals to the corresponding half of $\partial_{2} B$ (which has end points $Q$ and $S$ ). Now extend $h$ to a smooth homeomorphism from the whole of $\partial_{2} A$ to the whole of $\partial_{2} B$, using the involutions $j$ and $\sigma$, and then to a smooth homeomorphism from $\partial_{1} A$ to $\partial_{1} B$ by pulling back via $q$ and $\sigma \circ g$. This gives a smooth $h: \partial A \rightarrow \partial B$ equivariant with respect to the boundary data. But any smooth homeomorphism of boundaries of annuli extends to a quasiconformal homeomorphism $h$ of the interiors: this follows 
at once from the corresponding result for discs [2], since an annulus can be converted into a disc by cutting along any smooth path joining the inner boundary to the outer.

Let $\mu$ denote the complex dilatation $(\partial h / \partial \bar{z}) /(\partial h / \partial z)$ of $h$. By standard theory of quasiconformal maps [2] $\mu$ is of class $L^{\infty}$ (bounded almost everywhere) and $\|\mu\|_{\infty}<1$.

We shall abuse notation to the extent of denoting by $g$ not only the $2: 2$ correspondence on $B$ defined in Section 4.1, but also the correspondence $g_{A}=h \circ g_{B} \circ h^{-1}$ on $A$ obtained by transporting $g\left(=g_{B}\right)$ from $B$. Thus

$$
\left\{q: \partial_{1} A \rightarrow \partial_{2} A\right\}=\left\{j \circ g: \partial_{1} A \rightarrow \partial_{2} A\right\}
$$

and this degree two map has covering involution

$$
\left\{i: \partial_{1} A \rightarrow \partial_{1} A\right\}=\left\{g: \partial_{1} A \rightarrow \partial_{1} A\right\} .
$$

Moreover the Beltrami differential $\mu$ on $A$ is preserved by $g=g_{A}: A \rightarrow A$, in the sense that $g^{*} \mu=\mu$. Since $g_{B}^{*}$ is the identity, $g_{B}$ being holomorphic, this follows at once from the fact that

$$
g_{A}^{*} \mu=\left(h^{-1}\right)^{*} \circ\left(g_{B}\right)^{*} \circ h^{*}(\mu)
$$

4.3. Constructing the correspondence at the combinatorial/topological level. We first glue together $U$ and a second copy $U^{\prime}$ of $U$, via the boundary involution $j$, to obtain a sphere $U \cup U^{\prime}$, equipped with an involution, which we also denote $j$, exchanging $U$ with $U^{\prime}$ and restricting to the original $j$ on the common boundary. Inside $U^{\prime}$ we have a simply-connected subdomain $V^{\prime}$, corresponding to $V \subset U$. Let $q^{\prime}=j \circ q \circ j: V^{\prime} \rightarrow U^{\prime}$ denote the quadratic map corresponding to $q: V \rightarrow U$ and $A^{\prime}$ denote the annulus $U^{\prime}-V^{\prime}$. To define a $2: 2$ correspondence $f$ on $U \cup U^{\prime}$ we fit together:

- $q: V \rightarrow U$ (a $2: 1$ correspondence);

- $\left(q^{\prime}\right)^{-1}=j \circ q^{-1} \circ j: U^{\prime} \rightarrow V^{\prime}$ (a $1: 2$ correspondence);

- $j \circ i: V \rightarrow V^{\prime}$ (a $1: 1$ correspondence), and

- $j \circ g: A \rightarrow A^{\prime}$ (a $2: 2$ correspondence),

where $g: A \rightarrow A$ is the $2: 2$ correspondence constructed in Section 4.2 above. We remark that conjugation by the involution $j$ sends $f$ to $f^{-1}$. Thus $j$ is a timereversing symmetry of $f$.

Using the boundary data identities of Section 4.2 it is a straightforward exercise to check that the restrictions of $f$ defined above fit together to define a continuous $2: 2$ correspondence $f$ on the whole Riemann sphere. The next step is to identify the space of grand orbits of mixed iteration of $f$ and $f^{-1}$ on the complement $\Omega$ of $K(q) \cup K\left(q^{\prime}\right)$. Let $A / \sim$ denote the quotient space obtained from the (closed) annulus $A$ by applying the equivalence relation $g$ on $A$ and the equivalence relation $\langle g, j\rangle$ on $\partial A$, and let $\Delta / \approx$ denote the quotient space obtained from $\Delta$ (Figure 2 ) by identifying $l$ with $\chi l, m$ with $\sigma m$ and $n$ with $\rho n$.

Lemma 2. The grand orbit space of the correspondence $f$, acting on $\Omega$ by arbitrary combinations of forward and backward iteration, is homeomorphic to $A / \sim$ and hence to $\Delta / \approx=\Omega(G) / G$.

Proof. We first observe that if $z \in A$, then $g(z)(\subset A)$ and $j(z)\left(\in A^{\prime}\right)$ lie on the grand orbit of $z$ under $f$. This is because $g(z) \subset f^{-1} \circ f(z)$ and $j(z) \subset f^{-1} \circ f \circ f^{-1}(z)$. Now we must show that the grand orbit of any point in $\Omega$ meets $A$ in a single $g$-orbit. 
Clearly for each $z \in V \cap \Omega$ there is a unique positive integer $n$ such that $q^{n}(z) \in A$ and for each $z^{\prime} \in V^{\prime} \cap \Omega$ there is a unique positive integer $n$ such that $\left(q^{\prime}\right)^{n}\left(z^{\prime}\right) \in A^{\prime}$, and hence $j \circ\left(q^{\prime}\right)^{n}\left(z^{\prime}\right) \in A$ (the claim of uniqueness needs qualification if $q^{n}(z) \in \partial A$ or $\left(q^{\prime}\right)^{n}\left(z^{\prime}\right) \in \partial A^{\prime}$ but it is not hard to make the appropriate changes). However, in order to show that $q^{n}(z)$ and $j \circ\left(q^{\prime}\right)^{n}\left(z^{\prime}\right)$ are the only points of the grand orbits of $z$ and $z^{\prime}$ to lie in $A$, we need to check that no other points of $A$ can be reached by mixing iterations of $q$ and $q^{\prime}$ with the other branch, $j \circ i$, of $f$ (which, we recall, carries $V$ bijectively to $V^{\prime}$ ). It will suffice to verify that for any $z \in V$,

$$
j \circ\left(q^{\prime} \circ(j \circ i)\right)(z)=q(z) .
$$

However, $j \circ q^{\prime} \circ j=q$ and $q \circ i=q$, so we are done.

4.4. Making the correspondence holomorphic. Since $\partial U$ is smooth and the boundary involution $j: \partial U \rightarrow \partial U$ is smooth, the complex structure on $U$ extends to a complex structure on the sphere $U \cup U^{\prime}$. (It first descends to a complex structure on the quotient $U / j$ and then lifts to the double cover $U \cup U^{\prime}$.)

Consider the Beltrami differential $\mu$ on $A$ provided by the complex dilatation of the quasiconformal homeomorphism $h: A \rightarrow B$ (Lemma 1). We may extend $\mu$ to $q^{-1}(A)$ by setting its value there to be that of the pull-back $q^{*} \mu$, and we may extend it to $A^{\prime}$ by defining its value there to be that of $j^{*} \mu$. Indeed by repeatedly pulling back using $q^{*}$ and $\left(q^{\prime}\right)^{*}$ we may extend $\mu$ to $U-K=\bigcup q^{-n}(A)$ and $U^{\prime}-K^{\prime}=\bigcup\left(q^{\prime}\right)^{-n}\left(A^{\prime}\right)$, where $K$ and $K^{\prime}$ are the filled Julia sets of $q$ and $q^{\prime}$ respectively. Finally by defining it to be zero on $K \cup K^{\prime}$ we may extend $\mu$ to an $L^{\infty}$ Beltrami differential $\mu$ on the whole of the Riemann sphere. Since $\|\mu\|<1$, we may now apply the Measurable Riemann Mapping Theorem [1, 2] and deduce that there exists a quasiconformal homeomorphism $\phi: \hat{\mathbb{C}} \rightarrow \hat{\mathbb{C}}$ with complex dilatation $\mu$. But $f^{*} \mu=\mu$, since $g^{*} \mu=\mu, j^{*} \mu=\mu$ and $q^{*} \mu=\mu$ on the appropriate regions. Thus, by the chain rule, $\phi \circ f \circ \phi^{-1}$ is holomorphic, except possibly at branch points. But the latter are removable and hence the $2: 2$ correspondence $\phi \circ f \circ \phi^{-1}$ is holomorphic everywhere. Since $\mu$ vanishes on $K \cup K^{\prime}$, and is the complex dilatation of $h$ on $A$, the correspondence is a mating (in the sense of the definition in Section 3) of the quadratic map $q$ and the representation $r$ used in its construction, with the union of the images of $\Delta$ under $C_{2} * C_{3}$ as the fundamental domain $D$ for the action of $\chi$ on $\Omega(G)$, and with the grand orbit under $f$ of the image (under $h^{-1}$ ) in $A$ of the curve $l \subset \partial \Delta$ as the set of curves $\mathcal{C}$ such that $\Omega-\mathcal{C}$ is conformally homeomorphic to $D$. In Figure 4, where the coordinates have been chosen so that $j$ is the map $z \rightarrow-z$, we illustrate the annuli $A$ and $A^{\prime}$, their first few pre-images under $q^{-1}$ and $\left(q^{\prime}\right)^{-1}$ respectively, and the intersection of $\mathcal{C}$ with these annuli and pre-images. Note that each $q^{-n}(A)$ is an annulus, regularly $2^{n}$-fold covering $A$ itself, and that the union of all the annuli $q^{-n}(A)$ and $\left(q^{\prime}\right)^{-n}\left(A^{\prime}\right)$, when cut along the set of curves $\mathcal{C}$, opens out to form a disc (containing the point $\infty$ ).

With the complex structure defined above, the correspondence $f$ has graph an analytic subvariety $\mathcal{S}$ of $\hat{\mathbb{C}} \times \hat{\mathbb{C}}$. Such a subvariety is algebraic, by Chow's Theorem [6, 11, and therefore defined by a polynomial relation $p(z, w)=0$, quadratic in each of $z$ and $w$ since $f$ is a $2: 2$ correspondence. This completes the proof of Theorem 1. Moreover since the projection $(z, w) \rightarrow z$ of $\mathcal{S}$ to $\hat{\mathbb{C}}$ is a double cover with one double point, over the fixed point $P$ of $\rho$, and two branch points, over $T$ and the critical value of $q$, it follows by a calculation of Euler characteristic that $\mathcal{S}$ is of genus zero and hence, from the analysis in [3], that following a change in 


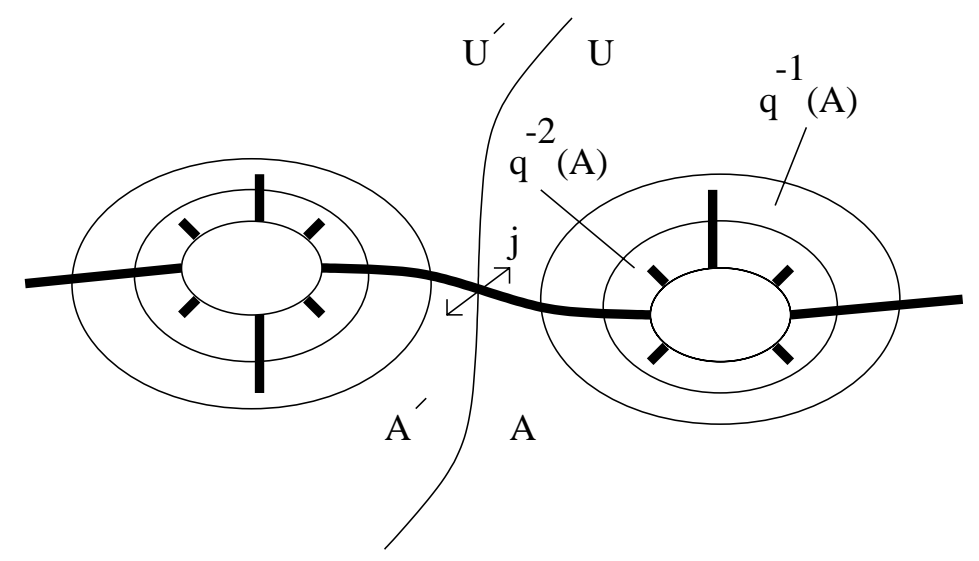

Figure 4. Pre-images of the annuli $A$ and $A^{\prime}$, and cut lines $\mathcal{C}$.

variable the relation $p(z, w)=0$ can be put in the form

$$
\left(\frac{a z+1}{z+1}\right)^{2}+\left(\frac{a z+1}{z+1}\right)\left(\frac{a w-1}{w-1}\right)+\left(\frac{a w-1}{w-1}\right)^{2}=3 k
$$

for some value of the (complex) parameters $a$ and $k$. When the correspondence is taken in this form, the (time-reversing) involution $j$ mapping the complementary subsets $U$ and $U^{\prime}$ of the complex plane bijectively to one another is $z \rightarrow-z$ (as in Figure 4).

In Figure 5 we display a computer plot of orbits of a correspondence $f$ in the family (1), with the values of the parameters $a$ and $k$ chosen such that the correspondence is one of the matings described in the theorem: indeed in this example the quadratic map is $z \rightarrow z^{2}$. The figure illustrates the grand orbits of the curves $n, l, m, \sigma m, \chi l, \rho n$ which make up the boundary of $\Delta$ in Figure 2, plotted to a certain depth, and a single grand orbit on $\partial K \cup \partial K^{\prime}$, plotted to a greater depth.

In [3] it was observed that all quadratic maps with connected Julia sets could be realised in the family of correspondences (1). The advantage of the present analysis is that the surgery approach shows that matings of all quadratic polynomials having connected Julia sets with all faithful discrete representations of $C_{2} * C_{3}$ having connected regular sets are realised in this family. We remark that computer experiment suggests we can go further: densely in the boundary of the space of representations of $C_{2} * C_{3}$ with connected regular set $\Omega$ lie the circle-packing representations, each still discrete and faithful but now having $\Omega$ a disjoint union of (round) discs. Each such representation is obtained by contracting an appropriate closed geodesic on the orbifold $\Omega / G$ to a point, and is characterised by a (rational) rotation number $\nu$ specifying the geodesic. Computer experiment strongly suggests that within the family (1) we can find a mating of each of these circle-packing representations with any quadratic map $z \rightarrow z^{2}+c$ such that $c$ does not lie in the $(1-\nu)$-limb of the Mandelbrot set, the latter being impossible for elementary combinatorial reasons. This topic will be explored elsewhere.

Analogous constructions can be made mating representations of $C_{p} * C_{q}$ with polynomial maps of degree $(p-1)(q-1)$ for arbitrary $p$ and $q$. See [5] for a related method which applies a generalisation of Klein's Combination Theorem. 


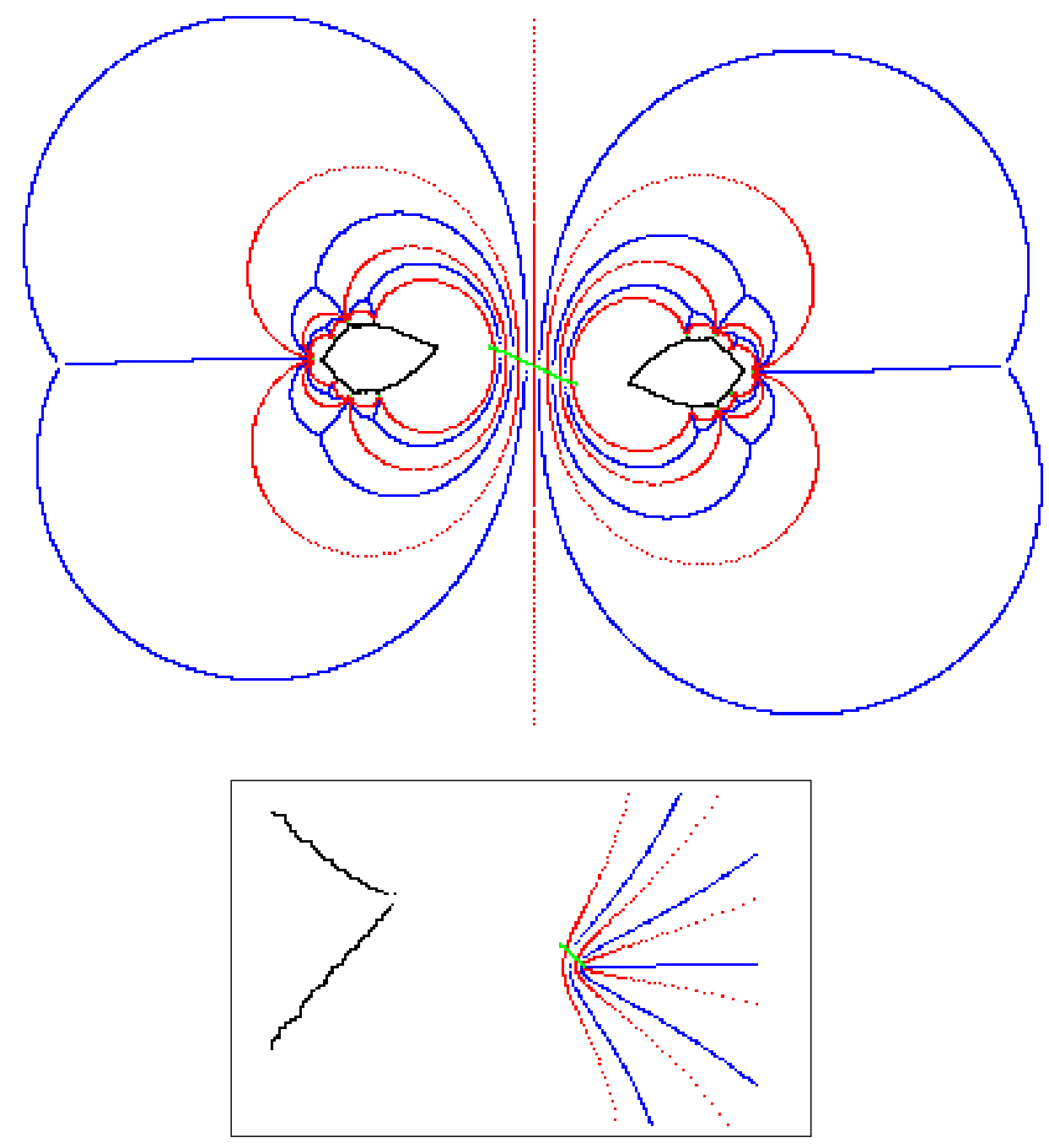

FiguRE 5. Orbits of correspondence (1) (and zoom around cusp on right), when $a=4.38+0.09 i$ and $k=0.91+0.04 i$.

\section{The Case When the QUadratic MaP has Disconnected Julia SET}

In the case considered so far, where $q$ has a connected Julia set, the construction of the mating is independent of the choice of equipotential made in order to define the domain $U$ (Section 1.1). When the Julia set is not connected, the critical value $c$ of $q$ lies in the basin of attraction of $\infty$ and the choice of equipotential used to define $U$ becomes significant, since the number $n$ such that $q^{n}(c) \in A=U-V$ is a topological invariant of the correspondence constructed. Thus when $c$ lies outside the Mandelbrot set, our initial data need to include not just the quadratic map $z \rightarrow z^{2}+c$ but also a choice of equipotential, which should lie outside the point $c$ so that both $U$ and $V$ are simply-connected and $A=U-V$ is an annulus. We can now construct both a $2: 2$ correspondence $f$ and a complex structure respected by it, just as we did in the case of connected Julia sets. This correspondence is no 
longer a mating of the quadratic map $q$ with the representation $r$ in the strict sense of the definition we gave earlier, since the presence in $\Omega$ of the critical value $c$ and its pre-images prevent us from obtaining a conjugacy to an action of $C_{2} * C_{3}$ in the way we did before. Nevertheless there is still a conformal homeomorphism between the orbit space $\Omega / f$ of the correspondence and that of the group $G=\langle\sigma, \rho, \chi\rangle$ on its regular set $\Omega(G)$, so it is clear how to recover the representation $r$ of $G$ from the correspondence. We also remark that when the representation $r$ is deformed to one lying on the boundary of moduli space, by contracting an appropriate geodesic on the orbit space to a point, certain restrictions come into play as to what positions are allowed for the critical value $c$. It seems likely that the effect is to exclude matings of the circle-packing representation of $C_{2} * C_{3}$ having rotation number $\nu$ with quadratic maps $z \rightarrow z^{2}+c$ having $c$ lying in the $(1-\nu)$-wake of $\mathcal{M}$. This question, like that towards the end of the previous section, will be further explored elsewhere.

\section{REFERENCES}

[1] L. Ahlfors and L. Bers, Riemann's mapping theorem for variable metrics, Annals of Math. 72 (1960), 385-404. MR 22:5813

[2] L. Ahlfors, Lectures on Quasiconformal Mappings, Van Nostrand 1966. MR 34:336

[3] S. Bullett and C. Penrose, Mating quadratic maps with the modular group, Inventiones Mathematicae 115 (1994), 483-511. MR 95c:58148

[4] S. Bullett and C. Penrose, Perturbing circle-packing Kleinian groups as correspondences, Nonlinearity 12 (1999), 635-672. MR 2000d:37054

[5] S. Bullett, A combination theorem for covering correspondences and an application to mating polynomial maps with Kleinian groups, QMW preprint 1999.

[6] W. Chow, On compact complex analytic varieties, Am. J. Math. 71 (1949), 893-914. MR 11:389f

[7] A. Douady and J. Hubbard, Itération des polynômes quadratiques complexes, C.R. Acad. Sci. Paris 294 (1982), 123-126. MR 83m:58046

[8] A. Douady and J. Hubbard, On the dynamics of polynomial-like mappings, Ann. scient. Éc. Norm. Sup. 18 (1985), 287-343. MR 87f:58083

[9] W. J. Harvey, Spaces of discrete groups, in Discrete groups and automorphic functions (edited by W. J. Harvey), Academic Press 1977, pp. 295-347. MR 58:16961

[10] B. Maskit, On Klein's combination theorem, Trans. AMS 120 (1965), 499-509. MR 33:274

[11] J.-P. Serre, Géométrie algébrique et géométrie analytique, Ann. Inst. Fourier 6 (1956), 1-42. MR 18:511a

[12] Lei Tan, Matings of quadratic polynomials, Erg. Th. and Dynam. Syst. 12 (1992), 589-620. MR 93h:58129

School of Mathematical Sciences, Queen Mary and Westfield College, University of London, Mile End Road, London E1 4NS, United Kingdom

E-mail address: s.r.bullett@qmw.ac.uk

Department of Mathematics, King's College, University of London, Strand, London WC2R 2LS, United Kingdom

E-mail address: bill.harvey@kcl.ac.uk 